\title{
Extra virgin olive oil polyphenolic extracts downregulate inflammatory responses in LPS-activated murine peritoneal macrophages suppressing NFKB and MAPK signalling pathways
}

\author{
A. Cárdeno, M. Sánchez-Hidalgo, M. Aparicio-Soto, S. Sánchez-Fidalgo \\ and C. Alarcón-de-la-Lastra*
}

Extra virgin olive oil (EVOO) is obtained from the fruit of the olive tree Olea europaea L. Phenolic compounds present in EVOO have recognized anti-oxidant and anti-inflammatory properties. However, the activity of the total phenolic fraction extracted from EVOO and the action mechanisms involved are not well defined. The present study was designed to evaluate the potential anti-inflammatory mechanisms of the polyphenolic extract (PE) from EVOO on LPS-stimulated peritoneal murine macrophages. Nitric oxide (NO) production was analyzed by the Griess method and intracellular reactive oxygen species (ROS) by fluorescence analysis. Moreover, changes in the protein expression of the proinflammatory enzymes, inducible nitric oxide synthase (iNOS), cyclooxygenase (COX)-2 and microsomal prostaglandin E synthase-1 (mPGES-1), as well as the role of nuclear transcription factor kappa B (NFKB) and mitogen-activated protein kinase (MAPK) signalling pathways, were analyzed by Western blot. PE from EVOO reduced LPS-induced oxidative stress and inflammatory responses through decreasing NO and ROS generation. In addition, PE induced a significant down-regulation of iNOS, COX-2 and mPGES1 protein expressions, reduced MAPK phosphorylation and prevented the nuclear NFKB translocation. This study establishes that PE from EVOO possesses anti-inflammatory activities on LPS-stimulated murine macrophages.

Received 9th January 2014

Accepted 8th March 2014

DOI: $10.1039 / c 4 f o 00014$ e

www.rsc.org/foodfunction

\section{Introduction}

Over the years, the so-called Mediterranean diet has become widely associated with improved health and well-being as well as protection against cardiovascular diseases and colon, breast and skin cancers. ${ }^{1}$ Epidemiological and clinical studies have demonstrated that low chronic disease risk observed in Mediterranean areas seems to be ascribed to high intake of fruits and oils from the olive tree Olea europaea L. $^{2}$ Among them, extra virgin olive oil (EVOO) is obtained from the fruits solely by mechanical or other physical means under conditions that do not lead to oil alteration. Within this context, the beneficial effect of EVOO consumption has been ascribed to non-polar lipids $^{3}$ or its high monounsaturated fatty acid (MUFA) content present in the major fraction of EVOO (98-99\%). ${ }^{4}$ However, EVOO also contains multiple minor components with important biological properties. Nowadays, it is clear that many of the beneficial effects of ingesting EVOO are due to its minor polyphenol compounds such as flavonoids, lignans

Department of Pharmacology, Faculty of Pharmacy, University of Seville, Profesor García González Street 2, 41012 Seville, Spain. E-mail: calarcon@us.es; Fax: +34 954556 074; Tel: +34954559877 (acetoxypinoresinol), secoiridoids (oleuropein-aglycone and ligstroside aglycone) and their hydrolysis products hydroxytyrosol (HT) and tyrosol (Ty), respectively, among others. These compounds have shown a broad spectrum of bioactive properties, including anti-oxidant, free radical scavenging, antiinflammatory and chemopreventive effects.

The anti-inflammatory effects of polyphenolic compounds have been largely described and attributed primarily to their capacity to scavenge and prevent both reactive oxygen species (ROS) and nitrogen species formation..$^{1,5}$ However, at cellular level, the activity of the total phenolic fraction extracted from EVOO and the plausible action mechanisms have not been completely described.

Macrophages are major inflammatory and immune effector cells, having a key role in the pathogenesis and development of inflammatory chronic diseases. ${ }^{6-8}$ The exposition to bacterial lypopolyssacharide (LPS), which acts as an endotoxin, drives the macrophages to an activated state where an excess of inflammatory mediators such as nitric oxide (NO) and prostaglandin $(\mathrm{PG}) \mathrm{E}_{2}$, as well as several pro-inflammatory cytokines, including tumor necrosis factor (TNF)- $\alpha$, interleukin (IL)-1 $\beta$, and IL-6, are produced. In addition, enzymes for example, cyclooxygenase (COX)-2 and inducible nitric oxide synthase (iNOS) are major 
effectors of the inflammation, which are well-regulated by mitogen-activated protein kinase (MAPK) family activation and nuclear transcription factor kappa B (NFкB) activation. ${ }^{9-11}$

Taking this background into account, the present study was designed to investigate the antioxidant and anti-inflammatory effects of the total phenolic fraction extracted from EVOO (PE) on LPS-stimulated murine macrophages. Also, we attempted to characterize the cellular mechanisms underlying PE possible anti-inflammatory activities, evaluating NO production, intracellular ROS and protein expression of COX-2, iNOS and mPGES-1. Moreover, we studied the role of MAPK and NFкB signalling pathways involved in these beneficial effects.

\section{Results}

\section{Effects of PE on cell viability in LPS-stimulated murine peritoneal macrophages}

After $18 \mathrm{~h}$, our data demonstrated that cell viability was not significantly reduced at concentrations up to $100 \mu \mathrm{g} \mathrm{mL} \mathrm{m}^{-1}$ for EVOO's PE on murine macrophages (data not shown). Based on these results, subsequent assays were carried out at concentrations of 25 and $50 \mu \mathrm{g} \mathrm{mL}{ }^{-1}$. The concentration of HT $(50 \mu \mathrm{M})$ was selected based on the HT concentration present in $50 \mu \mathrm{g}$ $\mathrm{mL}^{-1}$ of PE $(41.71 \mu \mathrm{M})$.

\section{PE inhibits LPS-induced intracellular ROS production in murine peritoneal macrophages}

Oxygen free radicals are suggested to be signalling messengers in the LPS-mediated inflammatory response. Thus, we tested the effects of EVOO's PE and HT on LPS-induced intracellular ROS production using the fluorescent probe DCFH-DA, which can be oxidized to the highly fluorescent compound DCF. As shown in Fig. 1, cells incubated using different treatments for $18 \mathrm{~h}$ exhibited significant decreases in intracellular ROS production; EVOO's PE (25 and $\left.50 \mu \mathrm{g} \mathrm{mL}^{-1}\right)(P<0.01$ vs. DMSO

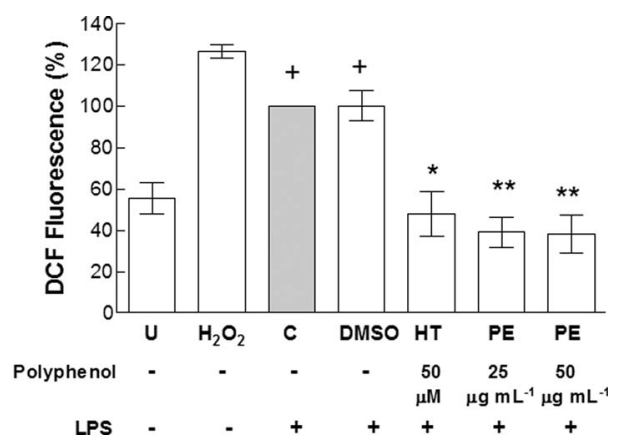

Fig. 1 Intracellular ROS generation reduced by PE and HT. Intraperitoneal murine isolated macrophages pretreated with LPS were incubated with PE (25 and $50 \mu \mathrm{g} \mathrm{mL}^{-1}$ ) or HT $(50 \mu \mathrm{M})$ for $18 \mathrm{~h}$. Then, cells were harvested and incubated with $10 \mu \mathrm{M}$ of DCFH-DA for $30 \mathrm{~min}$ at $37{ }^{\circ} \mathrm{C}$ in the dark. Results were expressed as mean fluorescence intensity obtained \pm S.E.M. $(n=3) .+P<0.05$ vs. untreated cells and $* P<0.05, * * P<0.01$ vs. LPS-DMSO treated control cells. $\mathrm{H}_{2} \mathrm{O}_{2}$ was used as the pro-oxidant positive control. control) and HT $(50 \mu \mathrm{M})(P<0.05$ vs. DMSO control $) . \mathrm{H}_{2} \mathrm{O}_{2}$ $(100 \mu \mathrm{M})$ was used as the pro-oxidant positive control.

$\mathrm{NO}_{2}{ }^{-}$production and iNOS expression are inhibited by PE in LPS-stimulated murine peritoneal macrophages

In macrophages and invading immune cells, the high amount of NO produced by iNOS in response to LPS and/or inflammatory cytokines plays a crucial role in inflammation and cytotoxicity. Thus we examined whether EVOO's PE inhibited NO release from activated macrophages. As shown in Fig. 2A, nitrite production as an indicator of NO production was substantially higher in cells treated with LPS than in those treated with the vehicle control. However both EVOO's PE and HT treatments significantly exhibited lower $\mathrm{NO}_{2}{ }^{-}$levels, $12.54 \%$ for $25 \mu \mathrm{g}$

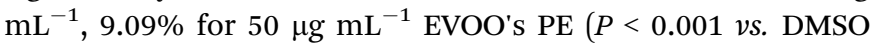
control) and $20.06 \%$ for HT treated cells $(P<0.001$ vs. DMSO control). This diminution of $\mathrm{NO}_{2}{ }^{-}$production suggested a possible down-regulation of iNOS enzyme activity. Thus, this hypothesis was assessed by measuring the iNOS protein levels. Immunoblotting analysis demonstrated that the decrease in $\mathrm{NO}_{2}{ }^{-}$levels was paralleled by a significant decrease in iNOS protein levels after incubation for $18 \mathrm{~h}$ using

A)

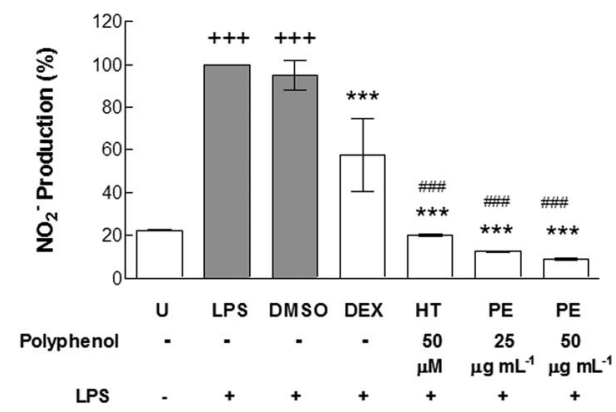

B)

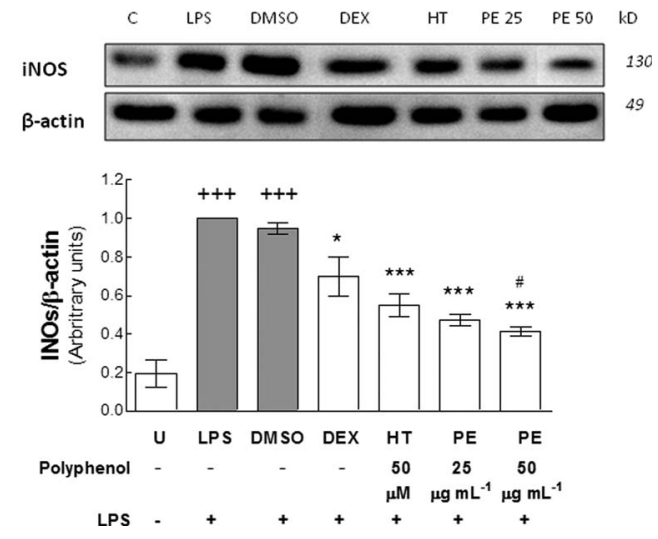

Fig. 2 Effect of PE and HT on release of nitrite generation. (A) Nitrite generation and (B) densitometric analysis of iNOS protein expression. The plots represent the band intensity and were measured using Image $J$ software. $\beta$-Actin served as an equal loading control for normalization. Each value represents the mean \pm S.E.M. for three independent experiments. $+++P<0.001$ vs. untreated cells, $* P<0.05, * * * P<0.001$ vs. LPS-DMSO treated control cells and \#P<0.05, \#\#\#P<0.001 vs. LPS-DEX. Dexamethasone $(1 \mu \mathrm{M})$ was used as the positive control. 
both treatments, EVOO's PE $\left(25\right.$ and $\left.50 \mu \mathrm{g} \mathrm{mL} \mathrm{m}^{-1}\right)(P<0.001 v s$. DMSO control) and HT $(50 \mu \mathrm{M})(P<0.001 v s$. DMSO control $)$ (Fig. 2B). Dexamethasone $(1 \mu \mathrm{M})$ was used as the antiinflammatory positive control. PE and HT treatments were significantly more efficient in inhibiting $\mathrm{NO}_{2}{ }^{-}$production and $\mathrm{PE}$ than dexamethasone treatment $(P<0.001 \mathrm{PE}$ or HT $v s$. DEX) (Fig. 2A). Moreover, statistical significant differences between data from cells treated with PE or DEX were found in iNOS protein levels $(P<0.05$ PE $v s$. DEX) (Fig. 2B).

\section{PE produced down-regulation in COX-2 and mPGES-1 overexpression induced by LPS}

We subsequently investigated the possible effects of EVOO's PE and HT on COX-2 inflammation-related enzyme. COX-2 protein expression was markedly induced by LPS treatment (Fig. 3A). However, a significant down-regulation on this proinflammatory protein expression was observed in cells treated with $50 \mu \mathrm{g} \mathrm{mL}{ }^{-1} \mathrm{PE}(P<0.05 v s$. DMSO control). Likewise, no statistical significant down-regulation from cells treated with

A)

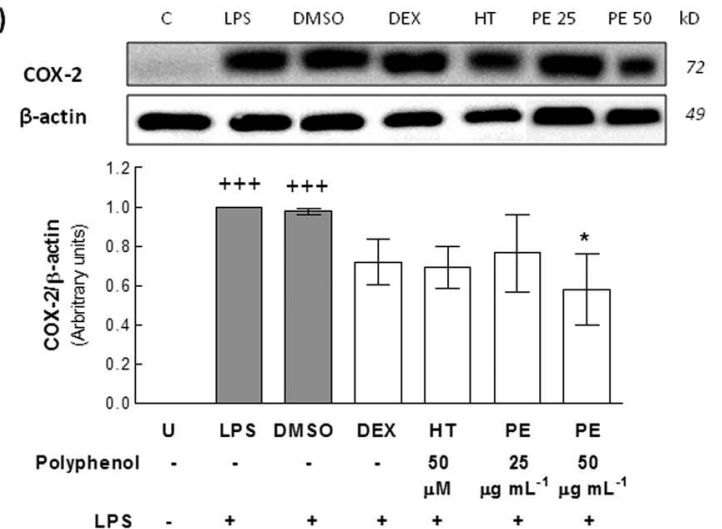

B)

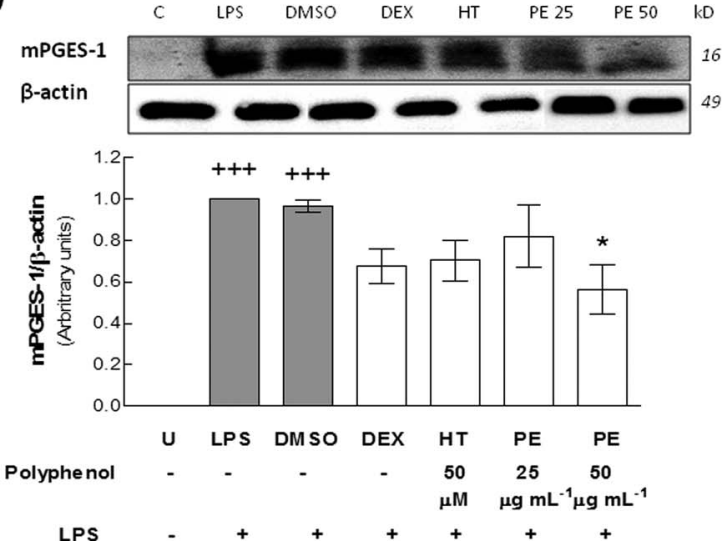

Fig. 3 PE inhibits COX-2 (A) and mPGES-1 (B) protein expression in murine intraperitoneal isolated macrophages. Cells were treated with PE (25 and $\left.50 \mu \mathrm{g} \mathrm{mL}^{-1}\right)$ or HT $(50 \mu \mathrm{M})$ for $18 \mathrm{~h}$ in the presence of LPS. As controls, cells were also treated with DMSO (solvent control) or left untreated in the absence of LPS. The plots represent the band intensity. $\beta$-Actin served as an equal loading control for normalization. Data are represented as mean \pm S.E.M. $+++P<0.001 \mathrm{vs}$. untreated cells and $* P<0.05$ vs. LPS-DMSO treated control cells. Dexamethasone $(1 \mu \mathrm{M})$ was used as the positive control.
$25 \mu \mathrm{g} \mathrm{mL}{ }^{-1}$ PE or $50 \mu \mathrm{M}$ HT on COX-2 expression was found. However $50 \mu \mathrm{M}$ HT seems to be more active than $25 \mu \mathrm{g} \mathrm{mL} \mathrm{m}^{-1} \mathrm{PE}$.

Similarly, LPS stimulation resulted in a marked expression of the mPGES-1 protein ( $P<0.001$ vs. untreated cells) (Fig. 3B). Though, $50 \mu \mathrm{g} \mathrm{mL}{ }^{-1} \mathrm{PE}$, but not $50 \mu \mathrm{M}$ HT treatments prior to LPS stimulation, resulted in significant inhibition of LPS-induced mPGES-1 protein expression $(P<0.05$ vs. DMSO control).

\section{PE reduces p38 and JNK phosphorylation in LPS-stimulated murine peritoneal macrophages}

To further explore the molecular mechanism underlying the anti-inflammatory effect of PE, we also determined its role in MAPK activation by Western blot analysis using phosphospecific MAPK antibodies. Cells were incubated in the absence or presence of different concentrations of PE before LPS stimulation. LPS induced the appearance of phosphorylated JNK and p38 $(P<0.05$ and $P<0.01$ vs. untreated cells $)$, whereas $\mathrm{PE}$ treatment demonstrated to inhibit significantly JNK and p38 activation $\left(25 \mu \mathrm{g} \mathrm{mL}{ }^{-1}, P<0.05\right.$ and $P<0.01 ; 50 \mu \mathrm{g} \mathrm{mL}^{-1}$, $P<0.05$ and $P<0.01$ vs. DMSO control, respectively) (Fig. 4). However, after $18 \mathrm{~h}$, HT treatment demonstrated the inability to inhibit the activation of JNK or p38 (Fig. 4).

\section{PE inhibits NF- $\kappa \mathrm{B}-$ mediated transcriptional activation and $\mathrm{I} \kappa \mathrm{B} \alpha$ degradation in murine peritoneal macrophages}

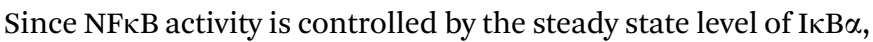
we further investigated $\mathrm{I} \kappa \mathrm{B}$ degradation in murine peritoneal
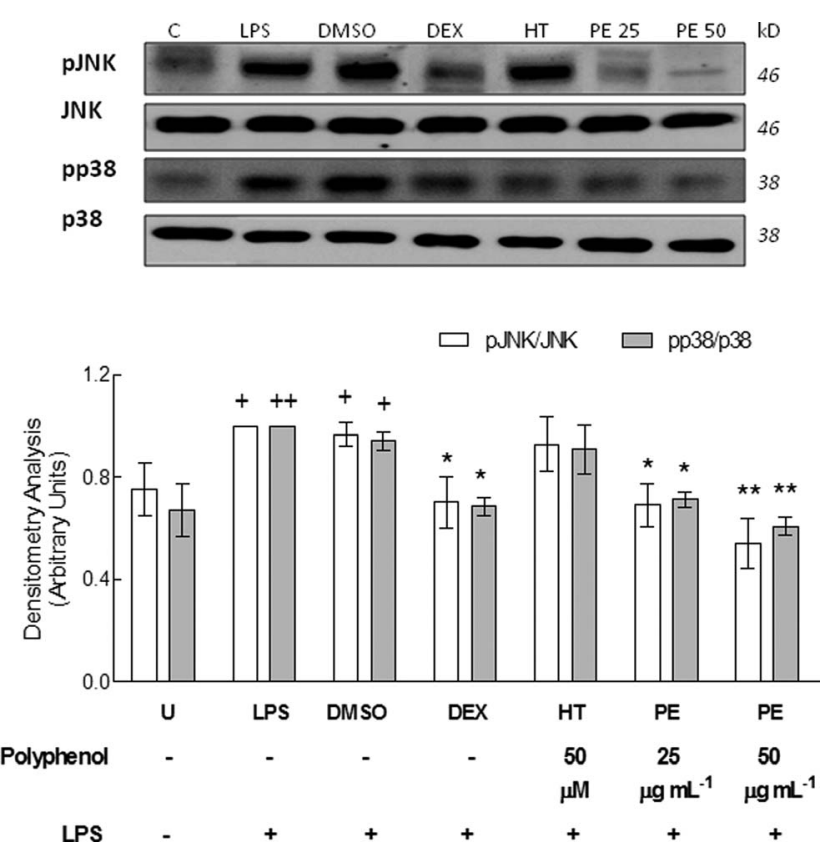

Fig. 4 Effects of PE and HT on pJNK and pp38 signalling pathways in murine intraperitoneal isolated macrophages. The results are representative of three independent experiments. Densitometry was performed following normalization to the control (JNK and p38 housekeeping genes, respectively). Data are expressed as the means \pm S.E.M. $+P<0.05$ and $++P<0.01$ vs. untreated cells and $* P<0.05$ and $* * P<0.01$ significantly different from LPS-DMSO treated control cells. Dexamethasone $(1 \mu \mathrm{M})$ was used as the positive control. 

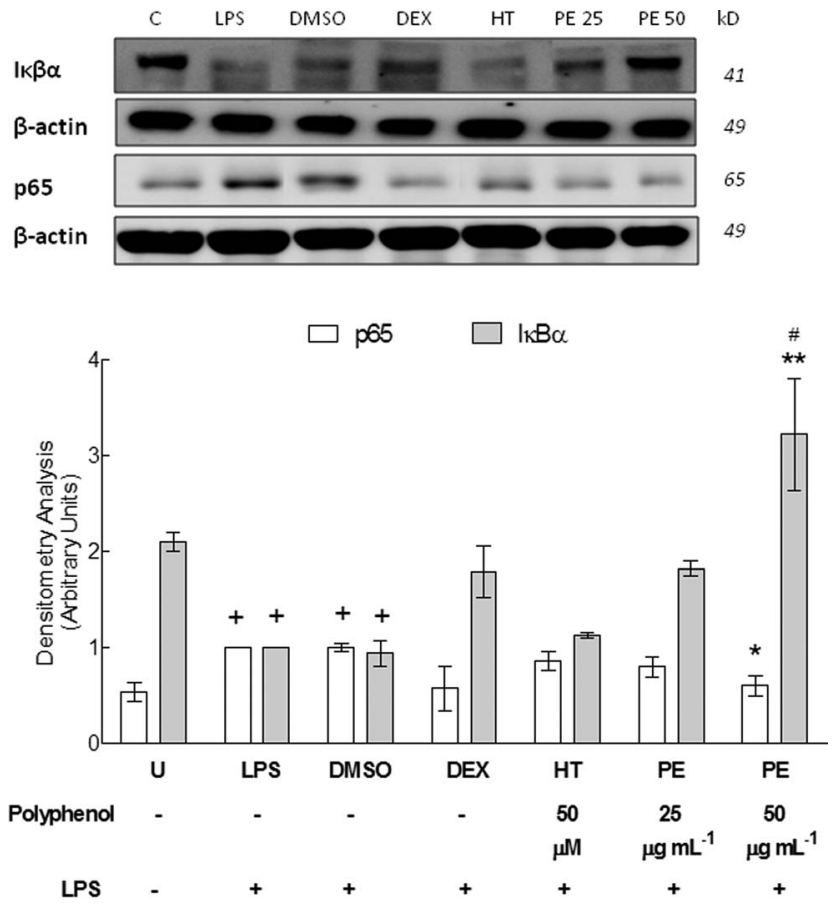

Fig. 5 PE treatment inhibits NFKB-mediated transcriptional activation and $1 \kappa B \propto$ degradation in murine intraperitoneal isolated macrophages. Results are representative of three independent experiments. Densitometry was performed following normalization to the control ( $\beta$-actin house-keeping gene). Data are expressed as the means \pm S.E.M. $+P<0.05$ vs. untreated cells, $* P<0.05$ and $* * P<0.01$ vs. LPSDMSO treated control cells and \#P<0.05 vs. LPS-DEX. Dexamethasone $(1 \mu \mathrm{M})$ was used as the positive control.

macrophages. LPS stimulation produced $\mathrm{I} \kappa \mathrm{B} \alpha$ degradation which was consistent with an up-regulation of NFKB-binding activity (Fig. 5) $(P<0.05$ vs. untreated cells). In contrast, PE treatment at $50 \mu \mathrm{g} \mathrm{mL} \mathrm{m}^{-1}$ prevented $\mathrm{I} \kappa \mathrm{B} \alpha$ degradation and subsequently ubiquitination $(P<0.01$ vs. DMSO control). Moreover, PE treatment caused a significant parallel inhibition of NFкB-mediated transcriptional activation, preventing the nuclear translocation level of p65 protein in murine macrophages, which was previously increased after LPS stimulation $(P<0.05 v s$. DMSO control). HT treatments did not produce any change in $\mathrm{I} \kappa \mathrm{B} \alpha$ or p65 protein expression. Dexamethasone $(1 \mu \mathrm{M})$ was used as the positive anti-inflammatory control in all protein assays. $50 \mu \mathrm{g} \mathrm{mL}{ }^{-1} \mathrm{PE}$ treatment was significantly more efficient in inhibiting $\mathrm{I} \kappa \mathrm{B} \alpha$ degradation than dexamethasone treatment $(P<0.05$ PE vs. DEX) (Fig. 5).

\section{Discussion}

Our findings have shown, for the first time, that PE from EVOO prevented the progression of cellular damage induced by LPS reducing the ROS levels and acting as an effective anti-oxidant.

Balance disruption of the intracellular reduction-oxidation state has been observed in activated macrophages, which leads to oxidative stress characterized by a major shift in the cellular redox balance and usually accompanied by ROS-mediated damage. Besides, ROS are capable of eliciting a variety of pathological changes, including the peroxidation of lipids, proteins, and DNA. Therefore, modulators of ROS production and ROS-induced signalling pathways, especially in macrophages, could represent potential targets for anti-inflammatory intervention. ${ }^{12}$ Our findings are in agreement with other in vitro studies where EVOO-isolated polyphenols i.e. HT or oleuropein showed strong anti-oxidant effects acting as powerful scavengers of free radicals in a similar range and higher than those tested in our study (50 to $100 \mu \mathrm{M}$ ). ${ }^{13,14}$

On the other hand, excess of NO acts as an intracellular messenger which modulates the formation of endogenous ROS that orchestrate the inflammatory responses. ${ }^{12,15}$ It has been reported that increased NO production in activated macrophages is due to increased levels of inducible iNOS expression, which in turn increase the transformation of $\mathrm{L}_{\text {-arginine to NO. }}{ }^{\mathbf{1 6}}$ In the present study we found that exposure of peritoneal macrophages to LPS resulted in a significant increase of nitrite/ nitrate levels as an indicator of NO production and an upregulation of iNOS expression and PE and HT treatments significantly were able to inhibit these effects. These results are in accordance with previous reports of Richard et al., (2011) where HT prevented the up-regulation of iNOS. Similar data were obtained by Zhang et al., (2009) in THP-1. ${ }^{17,18}$ Thus we suggest that PE and HT may decrease intracellular oxidant stress by direct ROS scavenging including reduced intracellular activation of redox-sensitive genes and subsequent downregulation of iNOS.

In addition, blocking of inflammatory enzymes like COX-2 or mPGES-1 represents important pharmacological tools for the treatment of inflammatory related diseases. ${ }^{19}$ mPGES- 1 is an efficient downstream enzyme for the production of $\mathrm{PGE}_{2}$ in LPSactivated macrophages, thus a selective inhibitor of mPGES-1 would be expected to inhibit $\mathrm{PGE}_{2}$ production induced by inflammation while sparing constitutive $\mathrm{PGE}_{2}$ production. ${ }^{20}$ Moreover, this enzyme is co-localized and functionally coupled with COX-2 which catalyzes the two sequential steps in the biosynthesis of PGs from arachidonic acid playing a critical role in the inflammatory response. ${ }^{21,22}$ The treatment of LPS-stimulated murine macrophages with PE, but not with $50 \mu \mathrm{M}$ of HT, produced down-regulation of both MPGES-1 and COX-2, indicating a potential dual action on these proteins and suggesting an inflammatory activity reduction. As such, the highest concentration used of PE $\left(50 \mu \mathrm{g} \mathrm{mL}^{-1}\right)$ contents about $50 \mu \mathrm{M}$ of HT; we might propose that this dual down-regulation implicated more olive oil polyphenols than HT.

MAPKs are a family of serine-threonine kinase enzymes which includes extracellular signal-regulated kinases ERKs-1 and -2, JNKs and p38 MAPKs. These proteins orchestrate the recruitment of gene transcription, protein biosynthesis, cell cycle control, apoptosis, and differentiation and allow cells to respond to oxidative stress and inflammatory stimuli. ${ }^{23}$ Similarly, the MAPK pathway is a critical axis essential for both induction and propagation of the inflammatory in the LPSactivated macrophage response. ${ }^{24}$ LPS was found to induce the appearance of phosphorylated JNK and p38, in contrast our results demonstrated that JNK and p38 activation were reduced by PE treatment, but not by HT. Although there are a few studies 
about the effects of olive oil polyphenols in the MAPK pathway, a recent study by our research group has also demonstrated that a diet made with EVOO and enriched with PE downregulated MAPK activation in an experimental ulcerative colitis model. ${ }^{25}$ The fact that we did not find any effect on MAPK regulation after HT treatment while PE exerted a significant inhibition in p38 and JNK-posporilation, even at $25 \mu \mathrm{g} \mathrm{mL}{ }^{-1}$, may significantly suggest a possible synergic effect between different compounds of PE.

$\mathrm{NF} \kappa \mathrm{B}$ signalling plays a key role in mediating inflammation and immune response through induction of pro-inflammatory cytokines, chemokines and other proteins. NFKB, as a dimeric transcription factor composed of p65 (RelA), RelB, c-Rel, NFкB1 (p50/p105) or NFאB2 (p52/p100), exists in the cytoplasm as an inactive complex with the inhibitory protein, I $\kappa \mathrm{B} \alpha$. When cells are challenged with pro-inflammatory stimuli, for example LPS, I $\mathrm{B} \alpha$ undergoes phosphorylation and subsequently ubiquitination, allowing NFKB to translocate to the nucleus. Consequently, $\mathrm{NF} \kappa \mathrm{B}$ binds to $\kappa \mathrm{B}$ enhancer elements present in the promoter region of many pro-inflammatory genes, for instance iNOS and COX-2. ${ }^{26,27}$ Our data showed that $18 \mathrm{~h}$ after LPSinduction PE significantly inhibited the degradation of $\mathrm{I} \kappa \mathrm{B} \alpha$ and blocked the translocation of p65 into the nuclei. This capacity of regulating NFKB genes was also described by Brunelleschi et al. (2007) who found that an EVOO extract particularly rich in phenolic compounds inhibited p50 and p65 NFкB translocation in monocytes and monocyte-derived macrophages (MDM) isolated from healthy volunteers. ${ }^{28}$ On the other hand, other research studies have shown that the anti-inflammatory activity of $\mathrm{HT}$ is mediated, at least in part, by $\mathrm{NF} \kappa \mathrm{B}$ signalling concentrations, however higher concentrations than $50 \mu \mathrm{M}$ were needed. For instance, Maiuri et al. (2005) suggested that HT at concentrations of $200 \mu \mathrm{M}$ might block the NFкB signalling pathway in J774 murine macrophages, ${ }^{29}$ as well as, Zhang et al. (2009) proposed that $100 \mu \mathrm{M}$, but not $50 \mu \mathrm{M}$ of HT reduced NFКB-p65 nuclear protein expressions in human monocytes THP-1. ${ }^{18}$ However, $50 \mu \mathrm{g} \mathrm{mL}{ }^{-1}$ of PE reduced NF $\kappa B$ translocation in LPS-stimulated macrophages suggesting that more polyphenols than HT are involved in the regulation of the $\mathrm{NF} \kappa \mathrm{B}$ pathway.

Together these data suggest that the total phenolic fraction extracted from EVOO may exert differential inhibitory effects in comparison with its isolated compounds, HT in this case, on the inflammatory response induced by LPS on murine macrophages.

\section{Experimental}

\section{Extraction and chemical characterization of EVOO- polyphenol extract}

EVOO (Olea europaea L, Picual variety, Aceite de las Valdesas, Córdoba, Spain) batch number 10/32 was used as the matrix to carry out PE. PE was obtained as Vazquez Roncero et al., (1997) ${ }^{30}$ described with some modifications. Fifty grams of oil was extracted with methanol : water $(80: 20, \mathrm{v} / \mathrm{v}, 125 \mathrm{~mL})$. The mixture was centrifuged at $5000 \mathrm{~g}$ for $1 \mathrm{~min}$ and sonicated for 15 min. After decantation, the methanolic extract was concentrated in a vacuum under a stream of nitrogen at $<35{ }^{\circ} \mathrm{C}$ until it reached a syrupy consistency; finally it was lyophilized and stored at $-80{ }^{\circ} \mathrm{C}$ until use. Quantitative and qualitative analyses of PE were performed according to COI/T20/29doc (International Olive Council) for olive oil based on direct extraction of the phenolic minor polar compounds from olive oil by means of a methanol solution and subsequent quantification by high-performance ternary gradient liquid chromatography (HPLC). After direct extraction of the phenolic minor polar compounds by means of a methanol solution, an aliquot of the supernatant phase was taken and filtered through a 0.45 mm PVDF filter, injected into the HPLC system equipped with a C18 reverse-phase column $(4.6 \mathrm{~mm} \times 25 \mathrm{~cm})$, a type Spherisorb ODS-2 $5 \mathrm{~mm}, 100 \AA$, with a spectrophotometric UV detector at $280 \mathrm{~nm}$ and an integrator. The content of the phenols was expressed in $\mathrm{mg} \mathrm{kg}^{-1}$ of oil and was calculated by measuring the sum of the areas of the related chromatographic peaks. The composition of the isolated PE is detailed in Table 1.

\section{Animals}

Male swiss mice (Harlan Interfauna Ibérica, Barcelona, Spain) weighing 20-30 g were placed in cages and maintained under constant conditions of temperature $\left(20-25{ }^{\circ} \mathrm{C}\right)$ and humidity (40-60\%) with a $12 \mathrm{~h}$ light/dark cycle and fed standard rodent chow (Panlab A04, Panlab, Seville, Spain) and water ad libitum throughout the experiment. All experiments were in accordance with the recommendations of the European Union (Directive of the European Counsel) regarding animal experimentation and followed a protocol observed by the Animal Ethics Committee of the University of Seville (approval no.: 86/609/EEC, 24 November 1986).

\section{Isolation and culture of murine peritoneal macrophages}

Mice were injected intraperitoneally with $1 \mathrm{~mL}$ of sterile thioglycollate medium (10\% w/v) (Scharlau, Barcelone, Spain). After 3 days, murine peritoneal macrophages were isolated as described previously by Cárdeno et al. (2013). ${ }^{31}$ Cells were treated with $5 \mu \mathrm{g} \mathrm{mL}{ }^{-1}$ LPS from $E$. coli (Sigma-Aldrich, St Louis, MO, US) in the presence or absence of PE, HT, or dexamethasone for $18 \mathrm{~h}$. HT (99\% pure) was purchased from Extrasynthese (Genay, France) and dexamethasone (99\% pure) from SigmaAldrich (St Louis, MO, US). Stock solutions were always freshly prepared in dimethylsulfoxide (DMSO) (Panreac, Barcelone, Spain) and diluted to a desired concentration directly in the culture medium. The final concentration of DMSO (Panreac, Barcelona, Spain) in all experiments was always $\leq 1 \%$ and it had not significantly influenced the cell response.

\section{Cell viability}

Cells seeded in 96-well plates $\left(1 \times 10^{5}\right.$ cells per well $)$ were incubated in the presence or absence of different PE concentrations for $18 \mathrm{~h}$. At the end of the exposure time, the effect on cell growth/viability was analyzed by sulforhodamine B (SRB) assay. Cell survival was measured as the percentage of absorbance compared with that obtained in control cells (non-treated cells). ${ }^{31}$ 
Table 1 Composition of the isolated EVOO polyphenolic extract using COI/T20/29doc

\begin{tabular}{|c|c|c|c|}
\hline Phenol name & $\begin{array}{l}\text { PE composition } \\
\operatorname{ppm}\left(\mathrm{mg} \mathrm{kg}^{-1}\right)\end{array}$ & $\begin{array}{l}\mu \mathrm{g} \text { phenol } \\
(50 \mu \mathrm{g} \mathrm{PE})\end{array}$ & $\begin{array}{l}\mu \mathrm{M} \text { phenol } \\
(50 \mu \mathrm{g} \text { PE per } \mathrm{mL})\end{array}$ \\
\hline Hydroxytyrosol & 45.00 & 6.43 & 41.71 \\
\hline Vanillic acid & 6.10 & 0.87 & 5.17 \\
\hline P-coumaric acid & 4.00 & 0.57 & 3.47 \\
\hline Decarboxymethyl oleuropein aglycone (dialdehyde) & 23.04 & 3.34 & 10.43 \\
\hline Pinoresinol & 15.70 & 2.24 & 6.25 \\
\hline Cinnamic acid & 6.80 & 0.97 & 6.54 \\
\hline Acetoxy-pinoresinol & 18.40 & 2.63 & 6.32 \\
\hline Oleuropein aglycone, aldehyde form & 88.20 & 12.60 & 39.37 \\
\hline Ligstroside aglycone, dialdehyde form & 56.60 & 8.09 & 26.61 \\
\hline Luteolin & 8.70 & 1.24 & 4.33 \\
\hline
\end{tabular}

\section{Measurement of intracellular ROS}

Intracellular ROS production was measured using 2,7-dichlorfluorescein-diacetate (DCFH-DA). DCFH-DA penetrates into the cells and is hydrolyzed by intracellular esterases to the nonfluorescent 2,7-dichlorofluorescein (DCFH), which can be rapidly oxidized to the highly fluorescent 2,7-dichlorofluorescein (DCF) in the presence of ROS. Peritoneal macrophages were seeded at $1 \times 10^{6}$ cells per well in 24 -well plates and incubated in the absence or presence of EVOO's PE or HT and 30 min later, cells were stimulated with LPS for $18 \mathrm{~h}$. The fluorescence intensity was measured as described previously by Cárdeno et al. (2013). ${ }^{31}$ After the incubation time, cells were treated with $10 \mu \mathrm{M} \mathrm{DCFH}-$ $\mathrm{DA}$ at $37{ }^{\circ} \mathrm{C}$ for $30 \mathrm{~min}$ and washed twice with PBS. The fluorescence intensity was measured with a plate reader (BioTek, Bad Friedrichshall, Germany) with an excitation wavelength of $485 \mathrm{~nm}$ and an emission wavelength of $538 \mathrm{~nm}$. The results were expressed as the intracellular ROS production percentage compared with LPS-DMSO control cells. $\mathrm{H}_{2} \mathrm{O}_{2}(100 \mu \mathrm{M})(30 \%$ pure) (Panreac, Barcelone, Spain) was used as the pro-oxidant positive control.

\section{Measurement of nitrite production}

Cells in a 24-well plate were untreated or treated with selected concentrations of PE, HT or dexamethasone and 30 min later they were stimulated with LPS for $18 \mathrm{~h}$. The amount of nitrite, as an index of NO generation, was determined as described previously by Cárdeno et al. (2013). ${ }^{31}$ The results were expressed as the nitrite production percentage compared with LPS control cells (stimulated untreated cells). $1 \mu \mathrm{M}$ dexamethasone 99\% (Sigma, St Louis, MO, USA) was used as the positive control.

\section{Isolation of proteins and immunoblotting detection}

Cells $\left(1 \times 10^{6}\right.$ cells per $\left.\mathrm{mL}\right)$ were untreated or treated with selected concentrations of PE, HT or dexamethasone, and stimulated with LPS for $18 \mathrm{~h}$. After incubation, the protein concentration was determined following Bradford's colorimetric method. ${ }^{32}$ Aliquots of supernatant containing an equal amount of protein $(20 \mu \mathrm{g})$ were evaluated to determine COX-2 iNOS, mPGES-1, IкB $\alpha$, p65, pJNK, JNK, pp38, and p38 proteins by Western blot as described by Cardeno et al. (2014). ${ }^{31}$ The signals were analyzed and quantified by Image Processing and Analysis in Java (Image J, Softonic). $1 \mu \mathrm{M}$ dexamethasone 99\% (Sigma, St Louis, MO, USA) was used as the positive control.

\section{Statistical analysis}

All values in the figures and text are expressed as arithmetic mean value \pm standard error (S.E.M). Data were evaluated with GraphPad Prism ${ }^{\circledR}$ Version 5.01 software. Comparison was done using one-way analysis of variance (ANOVA) followed by Tukey or Dunnett's test when appropriate. $P$ values of $<0.05$ were considered statistically significant. The figures shown are representative of at least three different experiments performed on different days.

\section{Conclusions}

In conclusion, this study establishes for the first time that the total phenolic fraction extracted from EVOO (PE) inhibits LPSinduced oxidative stress and inflammatory responses via direct downregulation of NO and ROS generation. These protective effects seem to be due to downregulation of iNOS, mPGES-1 and COX-2 expression via inhibition of MAPK activation and NF $\mathrm{B}$ signalling pathways.

Furthermore, our results suggest that HT plays an important role in the PE anti-oxidant and anti-inflammatory effects. Nevertheless other minor bioactive compounds present in PE such as oleuropein aglycone, tyrosol, pinoresinol or oleocanthal among others might contribute in synergy. In fact, previous reports have described olive oil polyphenols as a kind of natural product with antioxidant and anti-inflammatory properties. ${ }^{33-36}$ 


\section{Acknowledgements}

This work was supported by funds from the Spanish Ministerio de Ciencia e Innovación (AGL 2008-02475, AGL 2011-26949) and Junta de Andalucía (P-10AGR-6609; Proyecto de Excelencia). Authors gratefully acknowledge support from Postgraduate National of FPU and Inncorpora-Programa Torres Quevedo (INNCORPORA-PTQ) from Ministerio de Economía y Competitividad and Social European Fund. The authors also appreciate the assistance of the Center for Technology and Innovation Research, University of Seville (CITIUS). The authors declare no conflict of interest.

\section{Notes and references}

1 A. Cárdeno, M. Sánchez-Hidalgo and C. Alarcón-de-la-Lastra, An Up-date of Olive Oil Phenols in Inflammation and Cancer: Molecular Mechanisms and Clinical Implications, Curr. Med. Chem., 2013, 20, 4758-4776.

2 C. Alarcon de la Lastra, M. D. Barranco, V. Motilva and J. M. Herrerias, Mediterranean diet and health: biological importance of olive oil, Curr. Pharm. Des., 2001, 7, 933-950.

3 B. Bermudez, S. Lopez, A. Ortega, L. M. Varela, Y. M. Pacheco, R. Abia and F. J. Muriana, Oleic acid in olive oil: from a metabolic framework toward a clinical perspective, Curr. Pharm. Des., 2011, 17, 831-843.

4 H. C. Karantonis, N. Tsantila, G. Stamatakis, M. Samiotaki, G. Panayotou, S. Antonopoulou and C. A. Demopoulos, Bioactive polar lipids in olive oil, pomace and waste byproducts, J. Food Biochem., 2008, 32, 443-459.

5 M. V. Eberhardt, C. Y. Lee and R. H. Liu, Antioxidant activity of fresh apples, Nature, 2000, 405, 903-904.

6 T. Gui, A. Shimokado, Y. Sun, T. Akasaka and Y. Muragaki, Diverse roles of macrophages in atherosclerosis: from inflammatory biology to biomarker discovery, Mediators Inflammation, 2012, 2012, 693083.

7 A. F. Valledor, M. Comalada, J. Santamaria-Babi and A. Lloberas Celada, Macrophage proinflammatory activation and deactivation: a question of balance, Adv. Immunol., 2010, 108, 1-20.

8 G. Solinas, S. Schiarea, M. Liguori, M. Fabbri, S. Pesce, L. Zammataro, F. Pasqualini, M. Nebuloni, C. Chiabrando, A. Mantovani and P. Allavena, Tumor-conditioned macrophages secrete migration-stimulating factor: a new marker for M2-polarization, influencing tumor cell motility, J. Immunol., 2010, 185, 642-652.

9 G. Zhang and S. Ghosh, Molecular mechanisms of NFkappaB activation induced by bacterial lipopolysaccharide through Toll-like receptors, J. Endotoxin Res., 2000, 6, 453457.

10 J. Marks-Konczalik, S. C. Chu and J. Moss, Cytokinemediated transcriptional induction of the human inducible nitric oxide synthase gene requires both activator protein 1 and nuclear factor kappaB-binding sites, J. Biol. Chem., 1998, 273, 22201-22208.

11 J. R. Vane, J. A. Mitchell, I. Appleton, A. Tomlinson, D. Bishop-Bailey, J. Croxtall and D. A. Willoughby,
Inducible isoforms of cyclooxygenase and nitric-oxide synthase in inflammation, Proc. Natl. Acad. Sci. U. S. A., 1994, 9, 2046-2050.

12 D. Raederstorff, Antioxidant activity of olive polyphenols in humans: a review, Int J Vitam. Nutr. Res., 2009, 79, 152-165.

13 S. Granados-Principal, J. L. Quiles, C. Ramirez-Tortosa, P. Camacho-Corencia, P. Sanchez-Rovira and L. VeraRamirez, Hydroxytyrosol inhibits growth and cell proliferation and promotes high expression of sfrp4 in rat mammary tumours, Mol. Nutr. Food Res., 2011, 55, 117-1126.

14 D. Raederstorff, Antioxidant activity of olive polyphenols in humans: a review, Int. J. Vitam. Nutr. Res., 2009, 79, 152165.

15 G. C. Brown and V. Borutaite, Nitric oxide, mitochondria, and cell death, IUBMB Life, 2001, 52, 189-195.

16 A. K. Nussler and T. R. Billiar, Inflammation, immunoregulation, and inducible nitric oxide synthase, J. Leukocyte Biol., 1993, 54, 171-178.

17 N. Richard, S. Arnold, U. Hoeller, C. Kilpert, K. Wertz and J. Schwager, Hydroxytyrosol is the major anti-inflammatory compound in aqueous olive extracts and impairs cytokine and chemokine production in macrophages, Planta Med., 2011, 77, 1890-1897.

18 X. Zhang, J. Cao, L. Jiang and L. Zhong, Suppressive effects of hydroxytyrosol on oxidative stress and nuclear factor-kappaB activation in THP-1 cells, Biol. Pharm. Bull., 2009, 32, 578582.

19 B. Hinz, K. Brune and A. Pahl, Prostaglandin E(2) upregulates cyclooxygenase-2 expression in lipopolysaccharide-stimulated RAW 264.7 macrophages, Biochem. Biophys. Res. Commun., 2000, 272, 744-748.

20 M. Lazarus, C. J. Munday, N. Eguchi, S. Matsumoto, G. J. Killian, B. K. Kubata and Y. Urade, Immunohistochemical localization of microsomal PGE synthase-1 and cyclooxygenases in male mouse reproductive organs, Endocrinology, 2002, 143, 2410-2409.

21 M. Murakami, H. Naraba, T. Tanioka, N. Semmyo, Y. Nakatani, F. Kojima, T. Ikeda, M. Fueki, A. Ueno, S. Oh and I. Kudo, Regulation of prostaglandin E2 biosynthesis by inducible membrane-associated prostaglandin E2 synthase that acts in concert with cyclooxygenase-2, J. Biol. Chem., 2000, 275, 32783-32792.

22 I. Kudo and M. Murakami, Prostaglandin E synthase, a terminal enzyme for prostaglandin E2 biosynthesis, J. Biochem. Mol. Biol., 2005, 38, 633-638.

23 L. Munoz and A. J. Ammit, Targeting p38 MAPK pathway for the treatment of Alzheimer's disease, Neuropharmacology, 2010, 58, 561-568.

24 J. Chung, H. S. Lee, H. Y. Chung, T. R. Yoon and H. K. Kim, Salicylideneamino-2-thiophenol inhibits inflammatory mediator genes (RANTES, MCP-1, IL-8 and HIF-1alpha) expression induced by tert-butyl hydroperoxide via MAPK pathways in rat peritoneal macrophages, Biotechnol. Lett., 2008, 30, 1553-1558.

25 S. Sanchez-Fidalgo, A. Cardeno, M. Sanchez-Hidalgo, M. Aparicio-Soto and C. A. de la Lastra, Dietary extra virgin olive oil polyphenols supplementation modulates DSS- 
induced chronic colitis in mice, J. Nutr. Biochem., 2013, 24, 1401-1413.

26 P. P. Tak and G. S. Firestein, NF-kappaB: a key role in inflammatory diseases, J. Clin. Invest., 2001, 107, 7-11.

27 H. N. Lee and Y. J. Surh, Therapeutic potential of resolvins in the prevention and treatment of inflammatory disorders, Biochem. Pharmacol., 2012, 84, 1340-1350.

28 S. Brunelleschi, C. Bardelli, A. Amoruso, G. Gunella, F. Ieri, A. Romani, W. Malorni and F. Franconi, Minor polar compounds extra-virgin olive oil extract (MPC-OOE) inhibits NF-kappa B translocation in human monocyte/ macrophages, Pharmacol. Res., 2007, 56, 542-549.

29 M. C. Maiuri, D. De Stefano, P. Di Meglio, C. Irace, M. Savarese, R. Sacchi, M. P. Cinelli and R. Carnuccio, Hydroxytyrosol, a phenolic compound from virgin olive oil, prevents macrophage activation, Naunyn-Schmiedeberg's Arch. Pharmacol., 2005, 371, 457-465.

30 A. Vazquez Roncero, M. Janet del Valle and L. Janet del Valle, Componentes fenolicos de la aceituna. III, Polifenoles del aceite, Grasas Aceites, 1976, 27, 185-191.

31 A. Cárdeno, M. Sanchez-Hidalgo, M. Aparicio-Soto and C. Alarcón-de-la-Lastra, Unsaponifiable fraction from extra virgin olive oil inhibits the inflammatory response in LPSactivated murine macrophages, Food Chem., 2014, 147, 117-123.
32 M. M. Bradford, A rapid and sensitive method for the quantitation of microgram quantities of protein utilizing the principle of protein-dye binding, Anal. Biochem., 1976, 72, 248-254; H. Y. Kim, J. K. Kim, J. H. Choi, J. Y. Jung, W. Y. Oh, D. C. Kim, H. S. Lee, Y. S. Kim, S. S. Kang, S. H. Lee and S. M. Lee, Hepatoprotective effect of pinoresinol on carbon tetrachloride-induced hepatic damage in mice, J. Pharmacol. Sci., 2010, 112, 105-112.

33 S. Sanchez-Fidalgo, L. Sanchez de Ibarguen, A. Cardeno and C. Alarcon de la Lastra, Influence of extra virgin olive oil diet enriched with hydroxytyrosol in a chronic DSS colitis model, Eur. J. Nutr., 2013, 51, 497-506.

34 M. Scotece, R. Gomez, J. Conde, V. Lopez, J. J. Gomez-Reino, F. Lago, A. B. Smith and O. Gualillo, Further evidence for the anti-inflammatory activity of oleocanthal: inhibition of MIP1alpha and IL-6 in J774 macrophages and in ATDC5 chondrocytes, Life Sci., 2012, 91, 1229-1235.

35 M. Alirezaei, O. Dezfoulian, S. Neamati, M. Rashidipour, N. Tanideh and A. Kheradmand, Oleuropein prevents ethanol-induced gastric ulcers via elevation of antioxidant enzyme activities in rats, J. Physiol. Biochem., 2012, 68, 583-592.

36 E. Giner, I. Andujar, M. C. Recio, J. L. Rios, J. M. CerdaNicolas and R. Giner, Oleuropein ameliorates acute colitis in mice, J. Agric. Food Chem., 2011, 59, 12882-12892. 